Publ. RIMS, Kyoto Univ.

12 Suppl. (1977), 441-453.

\title{
Propagation at the Boundary and Reflection of Analytic Singularities of Solutions of Linear Partial Differential Equations I.
}

By

\author{
Pierre SCHAPIRA*
}

\begin{abstract}
Albstract
We study, microlocally, the non elliptic boundary value problems, with the help of the sheaf $\mathscr{C}_{N / X}$ of Kashiwara and Kawai. A hypothesis of "N-regularity" verified by several classes of preudo-differential operators allows us to obtain a theorem of reflection of analytic singularities analogous to that of Lax and Nirenberg.
\end{abstract}

\section{Introduction}

Our purpose is to extend to the analytic case, and to generalize, the theorem of Lax and Nirenberg [9] on the reflection of singularities. We will prove here a theorem similar to their's, but under a hypothesis of "N-regularity" satisfied in particular by micro-hyperbolic operators, as well as by the operators which are microlocally equivalent to the CauchyRiemann operator. This allows us to treat problems of reflection where, for the first case, many bicharacteristic strip are starting from the same point, and for the second, the bicharacteristic leaves are of dimension two.

The proof of the main theorem will be an adaptation of the proof of the general theorem of Kashiwara and Kawai on elliptic boundary value problems for systems [4], the remaining problem being to characterize N-regular operators. We only give here partial answers to this problem.

At the exception of $\S 5$ ("regularity II"), the results of this paper have been explained and published in "Seminaire Goulaouic-Schwartz, Décembre 1975."

Received July 12, 1976.

* Département de Mathématiques Université Paris-Nord Av. Jean Baptiste Clément 93430-Villetaneuse-France 


\section{$\S 1$. Preliminaries}

Our problem is of local type, near a boundary. Let then $\mathrm{M}$ be an open set of $\boldsymbol{R}^{n+1}$, with coordinates $(t, x)$, where $x=\left(x_{1}, \cdots, x_{n}\right), N$ be the hyperplane $\{t=0\}, X$ an open set of $\boldsymbol{C}^{n+1}$ which intersects $\boldsymbol{R}^{n+1}$ along $M, Y$ the complexification of $N$ in $X$.

Let $S_{N} * X, S_{Y}^{*} X, S_{M Y}^{*} X$ be the conormal sphere bundles of $N, Y, M$ in $X$, and $S_{N}{ }^{*} Y$ that of $N$ in $Y$. Let us recall that there are canonical isomorphisms $S_{M}{ }^{*} X \simeq i S^{*} M, S_{N}{ }^{*} Y \simeq i S^{*} N$. We will identify, when this will be without danger, a point in $T_{N} * X \backslash N$ and its image in $S_{N} * X$ (and similary for the other bundles) and we will spot a point $z^{*} \in S_{N} * X$ by its coordinates $(0, x, \tau, i \xi)$ that we prefer to write $(0, x, i \xi, \tau)$.

Let us denote, as in [4] by $p$ the projection of $S_{N}{ }^{*} X \backslash S_{Y} * X$ on $i S^{*} N$ :

$$
p:(0, x, i \xi, \tau) \rightarrow(x, i \xi)
$$

and by $\rho$ its restriction to $i S^{*} M \underset{M}{\times} N$ (recall that $i S^{*} M \underset{M}{\times} N=i S^{*} M \cap$ $\{t=0\})$. Let $M_{+}$be the open set $M \cap\{t>0\}, Q_{+}$the open set $S_{N} * X$ $\cap\{\operatorname{Re} \tau>0\}$ and similarly for $M_{-}$and $Q_{-}$. We denote by $\mathcal{O}$ the sheaf of holomorphic functions on $X, \mathscr{B}$ the sheaf of hyperfunctions on $M, \mathscr{C}$ the sheaf of microfunctions on $i S^{*} M$, and $\mathscr{B}_{N}$ and $\mathscr{C}_{N}$ the corresponding sheaves on $N$ and $i S^{*} N$. The singular support of a hyperfunction $f$ (we abbreviate it by s.s. $(f)$ ) is the support of the corresponding microfunction, and we say that $f$ is zero at some point $y^{*} \in i S^{*} M$ if $y^{*}$ does not belong to this singular support. We will also make use of the sheaf $\mathscr{L}$ of analytic pseudo-differential operators or rather of its restriction to $S_{N} * X$, and we refer to [10] for all those questions.

The sheaf $\mathscr{C}_{N \mid X}$ on $S_{N}{ }^{*} X$ is defined in [10] and used in an essential way by Kashiwara and Kawai in [4]. Let us recall its construction:

$$
\mathscr{C}_{N \mid X}=\mathcal{H}_{S_{N^{*} X}}^{n+1}\left(\pi_{N \mid X}^{-1} \Theta\right)^{a} \otimes \omega_{N}
$$

where $\omega_{N}$ is the orientation sheaf on $N$, a the antipodal map, and $\pi_{N \mid X}$ denotes the projection $X-N \cup S_{N} * X \rightarrow X$, the first space being endowed with the topology of the comonoidal transform (cf. [10] chapter 1). We then have canonical homomorphisms: 


$$
\Gamma_{N}(M, \mathscr{B}) \rightarrow \Gamma\left(S_{N}^{*} X, \mathscr{C}_{N \mid X}\right)
$$

$$
\begin{aligned}
& \Gamma_{\bar{M}_{+}}(M, \mathscr{B}) \rightarrow \Gamma\left(Q_{+}, \mathscr{C}_{N \mid X}\right) \\
& \mathscr{C}_{N|X|} \mid i S^{*} M \underset{M}{\times} N \rightarrow \mathcal{H}_{i S^{*}{ }_{M} \times N}^{0}(\mathscr{C}) \\
& \mathscr{C} \mid i S^{*} M \underset{M}{M} N \rightarrow \mathcal{H}_{i S^{*}{ }_{M} \times N}^{1}\left(\mathscr{C}_{N \mid X}\right)
\end{aligned}
$$

and Kashiwara [3] (cf. also [6]) has proved that homomorphisms (1.1) and $(1 \cdot 2)$ are injective, and homomorphisms $(1 \cdot 3)$ and $(1 \cdot 4)$ are, at least outside $S_{Y}{ }^{*} X$ injective. For $(1 \cdot 3)$ and $(1 \cdot 4)$, the only ones we need, this can be proved with the help of a (complex) quantized canonical transformation, but we do not give the proof here.

\section{§ 2. Division Theorems in $\mathscr{C}_{N \mid X}$}

A pseudo-differential operator $P$ of order $m$ is of Weierstrass type (in $D_{t}$ ) if it is written

$$
P=D_{t}{ }^{m}+\sum_{j=0}^{m-1} A_{j}\left(t, x, D_{x}\right) D_{t}^{j}
$$

the $A_{j}$ being pseudo-differential operators of orders $\leqq m-j$, with $\left[t, A_{j}\right]$ $=0$.

Let $x^{*}=(x, i \xi)$ belong to $i S^{*} N, P$ be a pseudo-differential operator of Weierstrass type of order $m$, defined near $p^{-1}\left(x^{*}\right)$ in $S_{N} * X$, and assume that the equation $P_{m}(0, x, i \xi, \tau)=0$ has a root of order $\mu$ at $\tau=\tau_{0}$. In this case there exists pseudo-differential operators of Weierstrass type, defined near $p^{-1}\left(x^{*}\right), E$ and $Q$, of respective order $m-\mu$ and $\mu$, with $P=E Q, E$ being invertible near $\left(0, x, i \xi, \tau_{0}\right)$ and the principal symbol of $Q$ having a root of order $\mu$ at this point (cf. [10] chapter 2). There exists also a similar decomposition of $P$ with $P=Q^{\prime} E^{\prime}$. We will say that they are Weierstrass decomposition of $P$.

The following lemma has been announced (in a more general formulation) by Kashiwara and Kawai [4] (cf. also [6]). It is the analogous in $\mathscr{C}_{N \mid X}$ of the Späth division theorem.

Lemma 1. Let $(x, i \xi)=x^{*}$ belong to $i S^{*} N$, $\tau_{0}$ be a root of order $\mu$ of the equation $P_{m}(0, x, i \xi, \tau)=0$. Let $z^{*}$ be the point $\left(0, x, i \xi, \tau_{0}\right)$ 
of $S_{N}{ }^{*} X$. Then for any $u \in\left(\mathscr{C}_{N \mid X}\right)_{2^{*}}$ there exists unique $v \in\left(\mathscr{C}_{N \mid X}\right)_{2^{*}}$, $\mathfrak{w}^{\prime}$ $\in\left(\mathscr{C}_{N}\right)_{x^{*}}(j=0, \cdots, \mu-1)$ solution of

$$
u=P v+\sum_{j=0}^{\mu-1} w_{j} \otimes \delta_{t}^{j}
$$

Lemma 3.5.2 of [10] chapter 2, permits to give a more global version of lemma 1 , but its formulation is quite general, and for sake of clearness we prefer to give the detailed proof in this context.

Lemma 2. Let $(x, i \xi)=x^{*}$ belong to $i S^{*} N, P$ be a pseudo-differential operator of Weierstrass type of order $m^{\prime}$ defined near $p^{-1}\left(x^{*}\right)$. Let $U$ be an open set of $S_{N}{ }^{*} X$ which contains exactly $m$ roots (counted with their multiplicities) of the equation $P_{m^{\prime}}(0, x, i \xi, \tau)=0$. Then for any $u \in \mathscr{C}_{N \mid X}\left(U \cap p^{-1}\left(x^{*}\right)\right)$ there exists unique $v \in \mathscr{C}_{N \mid X}\left(U \cap p^{-1}\left(x^{*}\right)\right)$ $w_{j} \in\left(\mathscr{C}_{N}\right)_{x^{*}}(j=0, \cdots, m-1)$ solution of:

$$
u=P v+\sum_{j=0}^{m-1} w_{j} \otimes \delta_{t}^{j}
$$

Proof. First we may assume, using a Weierstrass decomposition of $P$, that $m^{\prime}=m$. Let $\tau_{1}, \cdots, \tau_{r}$ be the distinct roots of the equation $P_{m}$ $\cdot(0, x, i \xi, \tau)=0$, with $(0, x, i \xi, \tau) \in U$, and $m_{1}, \cdots, m_{r}$ their multiplicities. Let us write $z_{j}^{*}$ for $\left(0, x, i \xi, \tau_{j}\right)$, and let, for any $j, P=E_{j} P_{j}$ be a Weïerstrass decomposition of $P, P_{j}$ being of order $m_{j}$, its principal symbol having a root of order $m_{j}$ at $\tau_{j}$.

The germ $u \in \mathscr{C}_{N \mid X}\left(U \cap p^{-1}\left(x^{*}\right)\right)$ being given, there exists by lemma 1 $v_{j} \in\left(\mathscr{C}_{N \mid X}\right)_{z^{*}} w_{i}^{j} \in\left(\mathscr{C}_{N}\right)_{x^{*}}$, solution of

$$
E_{j}^{-1} u=P_{j} v_{j}+\sum_{i=0}^{m_{j}-1} w_{i}^{j} \otimes \delta_{t}^{i}
$$

The operators $E_{j}$ being of Weierstrass type of order $m-m_{j}$, we can write

$$
E_{j}\left(w_{i}^{j} \otimes \delta_{t}^{i}\right)=\sum_{k=0}^{m-1} \widetilde{w}_{k}^{j} \otimes \delta_{i}^{k}
$$

with $\widetilde{w}_{k}^{j} \in\left(\mathscr{C}_{N}\right)_{x^{*}}$. Let us define $w_{k} \in\left(\mathscr{C}_{N}\right)_{x^{*}}$ by the equation

$$
\sum_{j=1}^{r} \sum_{i=0}^{m_{j}-1} E_{j}\left(w_{i}^{j} \otimes \delta_{t}^{i}\right)=\sum_{k=0}^{m-1} w_{k} \otimes \delta_{t}{ }^{k}
$$


We have, at the point $z_{l}{ }^{*}$ for $l \neq j$ :

$$
E_{j} v=E_{j} P_{j} P_{j}^{-1} v=P v^{\prime}
$$

So that at any point $z_{j}^{*}$ of $U, u$ can be written

$$
\begin{aligned}
& u=P v_{j}^{\prime}+\sum_{j=1}^{r} \sum_{i=0}^{m_{j}-1} E_{j}\left(w_{i}^{j} \otimes \delta_{t}{ }^{i}\right) \\
& u=P v_{j}{ }^{\prime}+\sum_{k=0}^{m-1} w_{k} \otimes \delta_{t}{ }^{k}
\end{aligned}
$$

This last equation defines $v_{j}^{\prime}$ on $U \backslash \bigcup_{k \neq j}\left\{z_{k}^{*}\right\}$ near $p^{-1}\left(x^{*}\right)$ and $v_{j}^{\prime}$ $=v_{l}^{\prime}$ on $U \backslash \bigcup_{k}\left\{z_{k}^{*}\right\}$ near $p^{-1}\left(x^{*}\right)$. This proves the existence of $v \mathscr{C} \in_{N \mid X}$ $\left(U \cap p^{-1}\left(x^{*}\right)\right)$ and $w_{j} \in\left(\mathscr{C}_{N}\right)_{x^{*}}$ satisfying equation $(2 \cdot 2)$.

Let us prove now the unicity of such a decomposition, and assume for that purpose that $P v=\sum_{j=0}^{m-1} w_{j} \otimes \delta_{t}{ }^{j}$. We proceed by induction on $r$. Let $Z_{1}=\left\{z_{1}^{*}\right\}, Z_{2}=\left\{z_{2}^{*}, \cdots, z_{r}{ }^{*}\right\}, P=P_{1} P_{2}$ a corresponding Weïrstrass decomposition.

We can find $v_{1}=\sum_{j=0}^{m_{1}-1} w_{j}^{1} \otimes \delta_{t}^{j}$ and $v_{2}=\sum_{j=0}^{m_{2^{\prime}}-1} w_{j}^{2} \otimes \delta_{t}^{j} \quad\left(\right.$ where $m_{2}^{\prime}=m-m_{1}$ ) such that

$$
\sum_{j=0}^{m-1} w_{j} \otimes \delta_{t}^{j}=P_{1} v_{2}+v_{1}
$$

(to see that, it is enough to write $w_{j} \otimes \delta_{t}^{j}$ as $D_{t}^{j}\left(w_{j} \otimes \delta_{t}\right)$ and to divide $D_{t}^{j}$ by $P_{1}$ ).

Then $v_{1}=P v-P_{1} v_{2}=P_{1} v_{1}{ }^{\prime}$, and lemma 1 applied at the point $z_{1}{ }^{*}$ implies $v_{1}=0$, so that $P v=P_{1} v_{2}$. As $P_{1}$ is invertible near $Z_{2}$ we deduce $v_{2}=P_{2} v$, and by the induction hypothesis, $v_{2}=0$, and then $w_{j}=0 \quad(j=0$, $\cdots, m-1)$, and $v=0$.

\section{§ 3. Definition and Properties of the Traces}

Let now $P$ be a differential operator with analytic coefficients on $M$, of order $m$, for which $N$ is non-characteristic. Let $f$ be a hyperfunction on $M_{+}$solution of $P f=0$. Let us recall [11], [7] how to define the traces of $f$ on $N$.

Let $\bar{f} \in \Gamma_{\bar{M}_{+}}(M, \mathscr{B})$ be an extension of $f$ supported by $\bar{M}_{+}: P \bar{f}$ is supported by $N$ and can be written in a unique way: 


$$
P \bar{f}=P g+\sum_{j=0}^{m-1} h_{j} \otimes \delta_{t}^{j}
$$

with $g \in \Gamma_{N}(M, \mathscr{B}), h_{j} \in \Gamma\left(N, \mathscr{B}_{N}\right)$,

The $\left(h_{j}\right)_{j}$, hyperfunctions on $N$, do not depend of the chosen extension: they are the traces of $f$ (the first one being $h_{m-1}$ ). This definition is not intrinsic but to say for example that the $p$ first traces of $f, h_{m-1}$, $\cdots, h_{m-p}$ are zero at some point $x^{*} \in i S^{*} N$ is invariant by analytic changes of coordinates.

If we look now at $P \bar{f} \in \Gamma_{N}(M, \mathscr{B})$ as an element of $\Gamma\left(\mathrm{S}_{N} * X, \mathscr{C}_{N \mid X}\right)$, we can, at any $x^{*} \in i S^{*} N$, apply lemma 2 and write in a unique way:

$$
P \bar{f}=u=P v+\sum_{j=0}^{m-1} w_{j} \otimes \delta_{t}^{j}
$$

with $w_{j} \in\left(\mathscr{C}_{N}\right)_{x^{*}}$. We will have $h_{j}=w_{j}$ at $x^{*}$.

If the $m$ traces of $f$ are analytic on $N, f$ will be analytic on $M_{+}$ near $N$ (by Holmgren's theorem). A theorem of Kashiwara permits to microlocalize this fact.

Lemma 3. Let $P$ be a differential operator of order $m$, the hypersurface $N$ being non-characteristic. Let $f$ be a hyperfunction on $M_{+}$solution of $P f=0$, and assume that the $m$ traces of $f$ are zero at $x^{*} \in i S^{*} N$.

Then $\rho^{-1}\left(x^{*}\right)$ does not intersect the closure in $i S^{*} M$ of the singular support of $f$.

Proof. We can choose the extension $\bar{f}$ of $f$ such that $P \bar{f}=\sum_{j=0}^{m-1} h_{j} \otimes \delta_{t}^{j}$. We then have $P \bar{f}=0$ in $\rho^{-1}\left(x^{*}\right)$; if we pick $\theta \in \boldsymbol{R}$ with $P_{m}(0, x, i \xi, i \theta) \neq 0$ (where $\left.(x, i \xi)=x^{*}\right)$, the point $(0, x, i \xi, i \theta)$ will not belong to the singular support of $\bar{f}$ ([10 theorem 2.1 .1 chapter 3$])$. It remains to apply a theorem of Kashiwara [3] (cf. also [6]): if $u$ belongs to $\Gamma_{\bar{M}_{+}}(M, \mathscr{B}$ ) and if $\left(0, x, i \xi, i \theta_{0}\right)$ belongs to the singular support of $u$ with $\xi \neq 0$, then $\forall \theta \in \boldsymbol{R},(0, x, i \xi, i \theta)$ belongs to the singular support of $u$.

Let us give an idea of the proof of Kashiwara's theorem. The main point is the injectivity of the homomorphism (1.4).

Let $u \in \Gamma_{\bar{M}}(M, \mathscr{B}), \widetilde{u}$ its image in $\Gamma\left(Q_{+}, \mathscr{C}_{N \mid X}\right)$. If $u$ is zero at $y^{*}$ 
$=\left(0, x, i \xi, i \theta_{0}\right) \in i S^{*} M \underset{M}{\times} N$, the "boundary value" of $\widetilde{u}$ in $H_{i S^{*}{ }_{M} N}^{1}\left(S_{N}{ }^{*} X\right.$, $\left.\mathscr{C}_{N \mid X}\right)$ will be zero at $y^{*}$, and then $\widetilde{u}$ is zero near $y^{*}$ in $Q_{+}$. But the sheaf $\mathscr{C}_{N \mid X}$ is, outside of $S_{Y}{ }^{*} X$, isomorphic to a sheaf of partially holomorphic microfunctions (the isomorphism being given by a Legendre quantized contact transformation [4]) and this implies that $\widetilde{u}$ is zero in $Q_{+} \cap p^{-1}$ $\cdot\left(x^{*}\right)$ (where $x^{*}=(x, i \xi)$ ). The boundary value of $\widetilde{u}$ will be zero on $\rho^{-1}\left(x^{*}\right)$, and by the injectivity of $(1 \cdot 4)$ this implies $u=0$ on $\rho^{-1}\left(x^{*}\right)$.

\section{§4. Regularity}

We will prove the main theorem under a hypothesis of " $N$-regularity" that we discuss now.

Definition. Let $N$ be an analytic hypersurface of $M, L=i S^{*} M \times \underset{M}{\times} N$, $y^{*} \in L \backslash i S_{N} * M$ and $P$ be a pseudo-differential operator defined near $y^{*}$. We say that $P$ is a $N$-regular at $y^{*}$ if $u \in\left(\Gamma_{L}(\mathscr{C})\right)_{y^{*}}, P u \in\left(\mathscr{C}_{N \mid X}\right)_{y^{*}}$ implies $u \in\left(\mathscr{C}_{N \mid X}\right)_{y^{*}}$

Let us assume for simplicity that $N$ is the hyperplane $\{t=0\}$ and recall [5] that a pseudo-differential operator $P$ of order $m$ defined near a point $\left(0, x_{0}, i \xi_{0}, i \theta_{0}\right)$ of $i S^{*} M$ is micro-hyperbolic in the direction $\mathrm{dt}$ if $N$ is non-characteristic and if all the roots in $\tau$ of the equation $P_{m}(t, x$, $i \xi, \tau)$ close to $i \theta_{0}$ are purely imaginary for $(t, x, \xi)$ near $\left(0, x_{0}, \xi_{0}\right)$.

Theorem 1. If $P$ is micro-hyperbolic in the direction dt at the point $y^{*} \in i S^{*} M \underset{M}{\times} N, P$ is $N$-regular at $y^{*}$.

Proof. We will use several properties of micro-hyperbolic operators proved by Kashiwara and Kawaï [5].

Let $u$ be a section of the sheaf $\mathscr{C}$ in a neighborhood of $y^{*}=\left(0, x_{0}\right.$, $\left.\mathrm{i} \xi_{0}, i \theta_{0}\right) u$ being supported by $L$, such that $P u$ belongs to $\mathscr{C}_{N_{i} X}$. We can assume that $P$ is of Weierstrass type of order $m$, the equation $P_{m}\left(0, x_{0}\right.$, $\left.i \xi_{0}, \tau\right)=0$ having a root of order $m$ at $\tau=i \theta_{0}$. Lemma 1 allows us to write: 


$$
P u=P v+\sum_{j=0}^{m-1} w_{j} \otimes \delta_{t}^{j}
$$

where $v \in\left(\mathscr{C}_{N \mid X}\right)_{y^{*}}, w_{j} \in\left(\mathscr{C}_{N}\right)_{x^{*}}\left(x^{*}=(x, i \xi)\right)$.

The operator $P$ being micro-hyperbolic in the direction $\mathrm{dt}$, we can [5 theorem 6.9] solve the equation

$$
P f=0, r(f)=(h)
$$

for any data $(h) \in\left(\mathscr{C}_{N}\right)_{x^{*}}^{m}, f$ being a microfunction near $y^{*}$, and $\gamma(f)$ denoting the restriction of $f$ and of its $m-1$ first derivatives in $t$ on $N$, which is meaningful because $N$ is non-characteristic for $P$ and soforth the projection $\rho$ is proper on the support of the microfunction $f$.

For the same reason we can make the product $Y(t) f$ of $f$ by the Heaviside function of $t$ : it is sufficient to take a hyperfunction $\bar{f}$ whose singular support is the closure in $i S^{*} M$ of the support of $f$, the image of $\bar{f}$ in $\mathscr{C}$ being $f$, and to restrict $Y(t) \bar{f}$ to the open set where $f$ was defined. To calculate $P(Y(t) f)$, where $P=D_{t}{ }^{m}+\sum_{0 \leqq j<m} A_{j}\left(t, x, D_{x}\right) D_{t}^{j}$, we remark that

$$
D_{t}(Y(t) f)=Y(t) D_{t} f+f(0, x) \otimes \delta_{t}
$$

and that

$$
A_{j}\left(t, x, D_{x}\right) Y(t) f=Y(t) A_{j}\left(t, x, D_{x}\right) f
$$

The first formula is trivial but the second is not. To prove it we can use the kernel $K_{j}(t, x, w)$ associated to $A_{j}\left(t, x, D_{x}\right)$ and an integral formula

$$
\int K(t, x, w) Y(t) \bar{f}(t, w) d w=Y(t) \int K(t, x, w) \bar{f}(t, w) d w
$$

(we do not give the detailed proof).

It is then easy to see that we can choose the data $(h)$ so that

$$
P(Y(t) f)=\sum_{j=0}^{m-1} w_{j} \otimes \delta_{t}^{j}
$$

we have now:

$$
P(u-v)=P(Y(t) f)
$$

and it is known [5 theorem 6.3] that the equation $P g=0$ has no non 
trivial solution supported by a half-space $\{t \geqq 0\}$ or $\{t \leqq 0\}$. This implies $u-v=Y(t) f$, and $f$ being supported by the half-space $\{t \leqq 0\}$ is zero.

\section{§5. Regularity $\mathbb{I I}$}

We will prove here the $N$-regularity of the operators micro-locally equivalent to the Cauchy-Riemann operator.

We denote by $\omega$ the canonical 1 -form on $i S^{*} M$, and by $\{$,$\} the$ Poisson bracket.

Theorem 2. Let $P$ be a pseudo-differential operator of order $m$ defined near $y^{*} \in i S^{*} M \underset{M}{\times} N$, of constant complex multiplicity, that is such that $P_{m}(t, x, i \xi, i \theta)=(q(t, x, i \xi, i \theta))^{r}$ with $d q \neq 0$. We assume that $d \operatorname{Req} \wedge d \operatorname{Im} q \wedge \omega \neq 0$, that $\{\operatorname{Req}, \operatorname{Im} q\}=O$ on the $\operatorname{set}\{(t, x, i \xi, i \theta)$ $q(t, x, i \xi, i \theta)=0\}$ and that $\{t, q\} \neq 0$. Then $P$ is N-regular at $y^{*}$.

Proof. We can perform a quantized real homogeneous canonical transformation to reduce the problem to the situation where $N=\{t=0\}$ and $P_{m}(t, x, \xi, \theta)=\left(\xi_{1}+i \theta\right)^{r}$. This is more or less classical, or as pointed out by Oshima, the same procedure as in [10 chapter 3 , theorem 2.2.1] gives the result.

Then theorem 5.2 .1 of [10chapter 2] reduces again the situation to the case where $P=D_{x_{1}}+i D_{t}$. In fact, using the notation $\widetilde{D}_{1}=D_{x_{1}}+i D_{t}$, we can write, by the Weierstrass preparation theorem for pseudo-differential operators

$$
P=E\left(\widetilde{D}_{1}^{r}+\sum_{0 \leqq j<r} A_{j} \widetilde{D}_{1}^{j}\right)
$$

where the $A_{j}$ are pseudo-differential operators of order $\leqq r-j-1$, which commute with $t+i x_{1}$, and $E$ is elliptic so that we can forget it.

Using a matrix notation, as in [10 p.436] we write

$$
\left(\widetilde{D}_{1} I_{r}-B\right) U \in\left(\mathscr{C}_{N \mid X}\right)^{r}
$$

where $I_{r}$ is the $r \times r$ unit matrix and $U$ is the $r$-column matrix with components $u, \widetilde{D}_{1} u, \cdots, \widetilde{D}_{1}^{r-1} u$. Then there exists an invertible matrix of pseudo-differential operators $A\left(t, x, D_{t}, D_{x}\right)$ such that 


$$
A\left(\widetilde{D}_{1} I_{r}-B\right) A^{-1}=\widetilde{D}_{1} I_{r}
$$

[cf. 10, chapter 2, demonstration of theorem 5.2.1]

We assume that $P=D_{x_{1}}+i D_{t}$ and look at $M$ as a partial complexification of $N$. Let us denote by $Z$ the complexification of $N$ with coordinates $\left(x_{1}+i t, x_{2}+i y_{2}, \cdots, x_{n}+i y_{n}\right)$. We have the embeddings $N \hookrightarrow M \hookrightarrow Z$. We denote by $\mathscr{B}_{h}$ the sheaf on $M$ of hyperfunction solutions of $P f=0$, that is of hyperfunctions holomorphic in $x_{1}+i t$, and by $\mathscr{C}_{h}$ the sheaf on $i S^{*} M$ of microfunctions which are solutions of the same equation. If $\tilde{\Lambda} \subset i S^{*} M$ is the characteristic set of $P$, we can identify $\tilde{\Lambda}$ with $S_{M} * Z$. We denote by $\Lambda$ the set $\tilde{\Lambda} \underset{M}{\times} N$ and identify if with its projection in $i S^{*} N$.

$$
\begin{aligned}
& \tilde{\Lambda}=\left\{(t, x, i \xi, i \theta) \in i S^{*} M \mid \xi_{1}=\theta=0\right\} \\
& \Lambda=\tilde{\Lambda} \cap\{t=0\} \simeq\left\{(x, i \xi) \in i S^{*} N \mid \xi_{1}=0\right\}
\end{aligned}
$$

Recall now that we have a canonical isomorphism [10 chapter 3 , theorem 2.2.5]

$$
\mathscr{C}_{h} \simeq \mathcal{H}_{S_{M} * Z}^{n-1}\left(\pi_{M \mid Z}^{-1} \mathcal{O}\right)
$$

and a canonical homomorphism [10 chapter 3 proposition 1.2.1]

$$
\boldsymbol{R} \Gamma_{S_{N^{*} Z} Z}\left(\pi_{N \mid Z}^{-1} \mathcal{O}\right) \mid S_{M K} * Z \underset{M}{*} N \rightarrow \boldsymbol{R} \Gamma_{S_{M^{4}{ }^{4} \times N}}\left(\pi_{M \mid Z}^{-1} \mathcal{O}\right)
$$

which induces the homomorphism

$$
\mathcal{H}_{S_{N^{*} Z}}^{n}\left(\pi_{N \mid Z}^{-1} \mathcal{O}\right) \mid S_{M} * Z \underset{M}{\times} N \rightarrow \mathcal{H}_{S_{M} * Z_{M} N}^{1}\left(\mathcal{F}_{S_{M}^{*}{ }^{*} Z}^{n-1}\left(\pi_{M \mid Z}^{-1}(\mathcal{O})\right)\right)
$$

that is

$$
\mathscr{C}_{N} \mid \Lambda \rightarrow \mathscr{K}_{\Lambda}{ }^{1}\left(\mathscr{C}_{h} \mid \tilde{\Lambda}\right)
$$

Homomorphism (5.4) is, by a quantized Legendre contact transform, the same as homomorphism (1.4) of $\S 1$ (with other notations), and soforth is injective. To explicit it, let us remark that the sheaf $\mathscr{C}_{N}$ being flabby it is determined by

$$
\mathscr{H}_{N}{ }^{n}\left(\mathcal{O}_{Z}\right) \rightarrow \mathscr{H}_{N}{ }^{1}\left(\mathcal{H}_{M}{ }^{n-1}\left(\mathcal{O}_{Z}\right)\right)
$$

that is

$$
\mathscr{B}_{N} \rightarrow \mathscr{H}_{N}{ }^{1}\left(\mathscr{B}_{h}\right)
$$

we can describe homomorphism (5.6) using the flabby resolution of $\mathscr{B}_{h}$ 
by $\mathscr{B}$ in the following way: to $g \in \mathscr{B}_{N}$ we associate the class modulo $P \mathcal{G}_{N}{ }^{0}(\mathscr{B})$ of $i\left(g \otimes \delta_{t}\right)$. (To prove it, use the example 3.2.1 of [10, chapter 1]).

Let now $v$ belong to $\mathscr{C}_{N}$. To say that the class of $v \otimes \delta_{t}$ modulo $P \mathcal{H}_{\Lambda}{ }^{0}(\mathscr{C} \mid \tilde{\Lambda})$ is unique in $\mathcal{H}_{\Lambda}{ }^{0}(\mathscr{C} \mid \tilde{\Lambda})$, means exactly that there exists no $u \in \mathcal{H}_{A}{ }^{0}(\mathscr{C} \mid \tilde{\Lambda})$ with $P u=v \otimes \delta_{t}$, and by lemma 1 it is equivalent to say there exists no $u \in \mathcal{H}_{\Lambda}{ }^{0}(\mathscr{C} \mid \tilde{\Lambda})$ (other than zero) such that $P u$ belongs to $\mathscr{C}_{N}{ }^{\prime} X$ and this proves the theorem.

\section{$\S 6$. Propagation at the Boundary and Reflection}

We use again the notations of $\S 3$.

Theorem 3. Let $P$ be a differential operator of order $m$ on $M$ for which $N$ is non-characteristic. Let $f$ be a hyperfunction on $M_{+}$ solution of $P f=0$. Let $(x, i \xi)=x^{*}$ be a point of $i S^{*} N$, and $Z^{-}, Z^{0}$, $Z^{+}$the set of points $(0, x, i \xi, \tau)$ of $S_{N} * X$ solution of $P_{m}(0, x, i \xi, \tau)=0$,

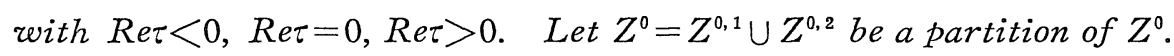
Assume:

a) at any point of $Z^{0,1}, P$ is $N$-regular.

b) the closure in $i S^{*} M$ of the singular support of $f$ does not intersect $Z^{0,1}$.

c) there are $m-p$ points (counted with their multiplicities) in $Z^{+}$ $\cup Z^{0,1}$.

d) the $p$ first traces of $f$ (that is $h_{m-1}, \cdots, h_{m-p}$ ) are zero near $x^{*}$. Then

- the $m$ traces of $f$ are zero near $x^{*}$

- the closure in $i S^{*} M$ of the singular support of $f$ does not intersect $\rho^{-1}\left(x^{*}\right)$.

Proof. The second part of the conclusion is a consequence of the first one and lemma 3 .

Let $\widetilde{Z}^{1}=Z^{+} \cup Z^{0,1}, \widetilde{Z}^{2}=Z^{-} \cup Z^{0,2}$.

There are $m-p$ points in $\widetilde{Z}^{1}$ and $p$ points in $\widetilde{Z}^{2}$.

Let $\bar{f} \in \Gamma_{\bar{M}_{+}}(M, \mathscr{B})$ be an extension of $f$ and $u$ the image of $P \bar{f}$ in 
$\mathscr{C}_{N \mid X}\left(p^{-1}\left(x^{*}\right)\right)$. Hypothesis $\left.d\right)$ implies

$$
u=P v+\sum_{j=0}^{m-p-1} w_{j} \otimes \delta_{t}^{j}
$$

where $v \in \mathscr{C}_{N \mid X}\left(p^{-1}\left(x^{*}\right)\right)$ and $w_{j} \in\left(\mathscr{C}_{N}\right)_{x^{*}}$.

At any point $z^{*}$ of $Q_{+}$, and in particular of $Z^{+}$, there exists $v$ $\in\left(\mathscr{C}_{N_{i} X}\right)_{z^{*}}$ with $u=P v$ : this is because $\Gamma_{\bar{M}_{+}}(M, \mathscr{B})$ is naturally sent in $\Gamma\left(Q_{+}, \mathscr{C}_{N \mid X}\right)$.

At every point $y^{*} \in Z^{0,1}$, hypothesis $b$ ) implies that $\bar{f}$ belongs to $\left(\Gamma_{L}(\mathscr{C})\right)_{y^{*}}$, where $L=i S^{*} M \underset{M}{\times} N$, and hypothesis $\left.a\right)$ implies then that $\bar{f}$ belongs to $\left(\mathscr{C}_{N \mid X}\right)_{y^{*}}$, because $P \bar{f} \in \Gamma_{N}(M, \mathscr{B}) \subset \Gamma\left(S_{N}{ }^{*} X, \mathscr{C}_{N \mid X}\right)$.

Let $U$ be an open set of $S_{N}{ }^{*} X$ which contains $\widetilde{Z}^{1}$ and does not intersect $\widetilde{Z}^{2}$. We can by the same argument as in the proof of lemma 2, find a common $v \in \mathscr{C}_{N \mid X}\left(U \cap p^{-1}\left(x^{*}\right)\right)$ with $u=P v$ in $U \cap p^{-1}\left(x^{*}\right)$.

We have in $U \cap p^{-1}\left(x^{*}\right)$ :

$$
P \bar{f}=u=P v \stackrel{m-p-1}{=} \sum_{j=0} \otimes \delta_{t}^{j}
$$

and $P_{m}(0, x, i \xi, \tau)$ has axactly $m-p$ roots in $U$. By lemma 2 we then have $w_{j}=0,(j=0, \cdots, m-p-1)$.

\section{$\S 7$. Some Remarks}

1) If the equation $P_{m}(0, x, i \xi, \tau)=0$ has no purely imaginary roots, we get a micro-local version of the Morrey-Nirenberg theorem [8], [11]. 2) If the operator $P$ has real simple characteristics, the bicharacteristic curves being transversal to $N$ (that is if the purely imaginary roots of $P_{m}(0, x, i \xi, \tau)=0$ are simple) we get in the analytic case, the theorem of reflexion of Lax and Nirenberg [9] (cf. also Chazarain's talk at this symposium). Of course we use the well known theorem of propagation along bicharacteristic curves [cf. 10 chapter 3 theorem 2.1.7].

3) Theorem 1 allows us to extend the Lax-Nirenberg theorem to situations where many bicharacteristic curves are starting from the same point (in $i S^{*} M$ ) (if $P$ is micro-hyperbolic at some point of $Z^{0,1}$, but not of constant real multiplicity). But we do not give any theorem of reflection between many bicharacteristics starting from the same point. 
Let us mention that the propagation of singularities at the boundary has also been studied by Kaneko for hyperbolic (not micro-hyperbolic) operators [2].

Theorem 2 allows us to extend the Lax-Nirenberg theorem to situations where the bicharacteristic leaves are of dimension two. We hope to treat the case of bicharacteristic leaves of any dimension (but transversal to $N$ ), that is to prove the $N$-regularity of the operators studied in [1].

4) Finally let us give an example of an operator which is not $N$-regular.

Let $M=\mathbb{R}^{2}$ with coordinates $\left(t, x_{1}\right), N$ the hyperplane $\{t=0\}, P$ the operator $D_{t}+i x_{1} D_{x_{1}}$ ("Lewy-Mizohata" operator). If $P$ were $N$-regular at the points $\left(0,0 ; \pm d x_{1}\right)$ it would imply by theorem 3 that any solution in $M_{+}$of the equation $P f=0$ would extend analytically across the boundary, which is not true.

\section{References}

[1] Bony J. M., Schapira P., Propagation des singularités analytiques pour les solutions des équations aux dérivées partielles, Ann. Inst. Fourier 26 (1976), (1) 81-140.

[2] Kaneko A., Singular spectrum of boundary values of solutions of partial differential equations with real analytic coefficients, Scientific Papers Coll. Gen. Educ. Univ. Tokyo $25 \mathrm{n}^{\circ} 2$ (1975), 59-68.

[3] Kashiwara M., Talks in Nice (1972).

[4] Kashiwa M., Kawaï T., On the boundary value problem for elliptic systems of linear differential equations, I and II, Proc. Japan Acad. 48 (1971), 712-715 and 49 (1973), 164-168.

[5] Kashiwara M., Kawaï, T., On microhyperbolic pseudo-differential operators I, J. Math. Soc. Japan, 27 (1975), 359-404.

[6] Kataoka K., A microlocal approach to general boundary value problems, these proceedings.

[7] Komatsu, H., Boundary values for solutions of elliptic equations, Proc. Intern. Conf. Funct. Analysis and Relat. Topics, Univ. of Tokyo Press, (1970), 107-121.

[8] Morrey C. B., Nirenberg L., On the analyticity of the solutions of linear elliptic systems of differential equations, Comm. Pure Appl. Math. 10 (1957), 261-290.

[9] Nirenberg, L., Lectures on linear partial differential equations, Regional Conferences series in Math., n. 17 Providence RI, 1973.

[10] Sato, M., Kawaï, T., Kashiwara, M., Hyperfunctions and pseudo-differential equations, Lecture Notes in Math., Springer 287, (1973), 265-529.

[11] Schapira, P., Hyperfonctions et problèmes aux limites elliptiques, Bull. Soc. Math. France 89 (1971), 113-141.

Note added in proof. We have now proved the $N$-regularity of a large class of pseudodifferential operators including those discussed in [1]. cf. Seminaire Goulaonic-Schwartz, Décembre 1976. 
